\title{
Calcified Meningioma Presenting as Syndrome of Inappropriate Antidiuretic Hormone in an Elderly Patient - A Rare Case Report
}

\author{
Shivam Khanna1, Yash Gupte ${ }^{2}$, Parth Godhiwala 3 , Sachin Agrawal ${ }^{4}$, Sunil Kumar ${ }^{5}$ \\ 1, 2, 3, 4, 5 Department of General Medicine, DMIMS, Sawangi, Wardha, Maharashtra, India
}

\section{INTRODUCTION}

In cancer patients, syndrome of inappropriate antidiuretic hormone secretion (SIADH) is the leading cause of hyponatremia. ${ }^{1}$ Hyponatremia is a serious disorder of dyselectrolytemia, linked with neurological symptoms that are life-threatening and it is the most common aetiology of tumour-related electrolyte imbalance. ${ }^{2}$ The tumours commonly causing hyponatremia of SIADH variety are lung, head-neck and breast tumours. SIADH has been documented in various malignancy types with numerous possible aetiologies apart from active malignancy. ${ }^{2,3}$ The aetiology distribution of SIADH in tumour patients are not completely known. Secondly, clinically, it is not clear which malignancy is most commonly associated with SIADH. ${ }^{3}$ Calcified meningioma presenting as SIADH had not been reported so far in the literature. Here a case of an elderly female with clinical features and brain imaging suggestive of meningioma associated with hyponatremia due to SIADH had been highlighted.

\section{PRESENTATION OF CASE}

An 80-year-old female patient was admitted to the medical intensive care unit of our hospital with complaints of drowsiness and vomiting for 2 days. The patient's relatives also gave a history of forgetfulness on and off. The patient was a known case of ischemic heart disease (IHD) with systemic hypertension on medication for 3 years. Her computerised tomography scan of the brain showed the right frontal lobe having a calcified meningioma. Her serum sodium levels of $123 \mathrm{mmol} / \mathrm{L}$, potassium of $3.5 \mathrm{mmol} / \mathrm{L}$, and serum osmolality of $259 \mathrm{mOsm} / \mathrm{kg}$. This case report highlights suspecting syndrome of inappropriate antidiuretic hormone secretion (SIADH) as the patient had hyponatremia.

On examination, the patient was drowsy and irritable. Her blood pressure (BP) was 140 / $80 \mathrm{mmHg}$, pulse of 110 beats per minute, normal jugular venous pressure and respiratory rate of 20 per minute. Saturation was $99 \%$ without 02 . There was no oedema in the lower limb. Her pupils reacted to light normally and papilloedema was not detected on fundus examination. The rest of the systemic examination was normal. There was no sign of dehydration. Neurological examination showed severe cognitive function impairment.

\section{DISCUSSION OF MANAGEMENT}

On routine investigations, her complete blood count showed haemoglobin as $9.5 \mathrm{gm}$ / dL, total leukocyte counts of 9,000 per cubic mm and platelets were normal. A renal function test was done which showed blood urea level of $14 \mathrm{mg} / \mathrm{dl}$, with a serum creatinine of $0.8 \mathrm{mg} / \mathrm{dl}$, serum sodium $(\mathrm{Na})+$ levels of $123 \mathrm{mmol} / \mathrm{L}$ and serum potassium $(\mathrm{K})+$ of $3.5 \mathrm{mmol} / \mathrm{L}$. Her hepatic profile was within normal limits. Her chest $\mathrm{X}$-ray was normal.
Corresponding Author: Dr. Shivam Khanna,

Room No. G-17,

Yashoda Boys Hostel, AVBRH, Sawangi (Meghe), Wardha, Maharashtra, India. E-mail: drshivamkhanna@gmail.com

DOI: $10.14260 / j e m d s / 2021 / 211$

How to Cite This Article:

Khanna S, Gupte Y, Godhiwala P, et al. Calcified meningioma presenting as syndrome of inappropriate antidiuretic hormone in an elderly patient - a rare case report. I Evolution Med Dent Sci 2021;10(13):986-987, DOI: 10.14260/jemds/2021/211

Submission 07-11-2020,

Peer Review 31-01-2021,

Acceptance 06-02-2021,

Published 29-03-2021.

Copyright (c) 2021 Shivam Khanna et al. This is an open access article distributed under Creative Commons Attribution License [Attribution 4.0 International (CC BY 4.0)] 
Given the low serum sodium levels, we investigated for urine osmolality which was $158 \mathrm{mOsm} / \mathrm{kg}$, serum osmolality which was $259 \mathrm{mOsm} / \mathrm{kg}$ and urine $\mathrm{Na}+$ was $21 \mathrm{mmol} / \mathrm{L}$ and urinary $\mathrm{K}+$ was 5.7. In the patient, hyponatremia associated with reduced osmolality with excessively increased urine osmolality, natriuresis, normal adrenal and thyroid function, with no finding of renal, cardiac and hepatic disease, makes the diagnosis of SIADH. Her serum uric acid level was 2.1. Her computerised tomography scan of brain revealed evidence of extra-axial lesion at high convexity. The frontal region showing dense calcification in centre. The lesion shows bare towards falx suggestive of meningioma (Figure 1). Her sodium was corrected with $3 \%$ normal saline infusion initially to correct the signs of severe hyponatremia. The patient's Glasgow Coma Scale (GCS) score improved gradually. The patient had partial improvement in cognition and blood chemistry became normal.

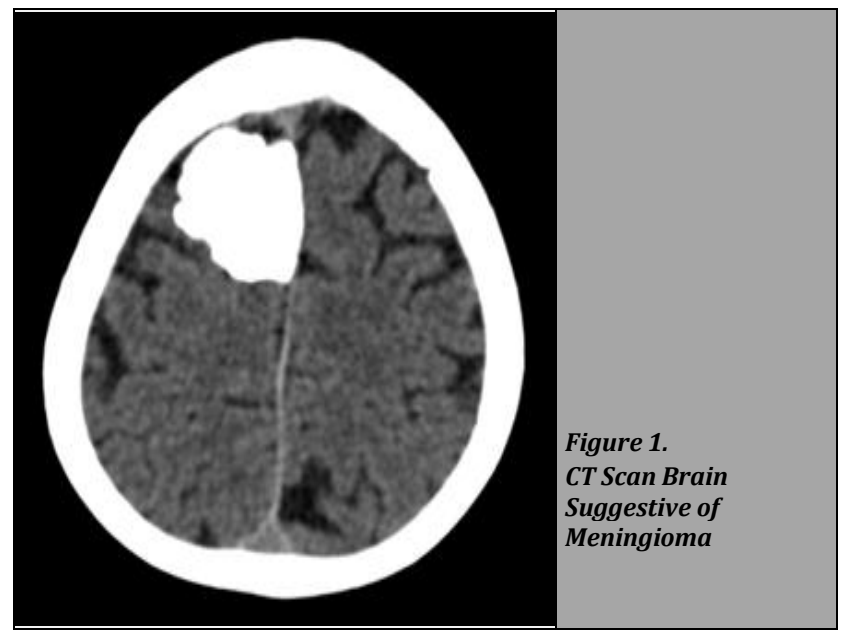

\section{DISCUSSION}

The disorder of SIADH is due to non-physiological release of hormone from the neurohypophysis due to low plasma osmolality. ${ }^{1}$ There is notable mortality and morbidity associated with hyponatremia in patients suffering from cancer. Stay in hospital is approximately twofold increase in admitted patients with hyponatremia of the category of moderate to severe type, as compared to normonatraemic patients. Other symptoms seen in hyponatremia like lethargy, confusion and headache affect the quality of life. Fluid restriction generally needed for SIADH treatment hampers the nutrition in many patients. Therefore, SIADH is a clinically significant and common finding in patients suffering from cancer. Moreover, in patients suffering from cancer, SIADH can be associated with drugs other than anticancer drugs, like antiepileptics or anti-depressants. Metastasis from the central nervous system and pulmonary can also cause SIADH. ${ }^{4}$

Hyponatremia in our patient was because of SIADH which was associated with meningioma, a relatively rare form in elderly patients. Essential diagnostic criteria for the diagnosis of SIADH are $\bullet$ serum $\mathrm{Na}+<135 \mathrm{mmol} / \mathrm{L}$; $\bullet$ urinary osmolality $>100 \mathrm{mOsm} / \mathrm{kg}$ during hypotonicity; $\bullet$ decreased plasma osmolality (< $275 \mathrm{mOsm} / \mathrm{kg})$; • clinical euvolemia; • no clinical signs of extracellular fluid contraction (e.g. no dry mucous membranes, or decreased skin turgor, orthostasis, tachycardia); - signs of expansion of extracellular fluid on clinical expansion not present (e.g. there is no ascites or oedema); normal adrenal and thyroid gland function determined by laboratory and clinical assessment; increased excretion of urinary sodium $>20 \mathrm{mmol} / \mathrm{L}$ with normal salt and water intake in diet. Supporting diagnostic criteria are • plasma hypouricemia $\bullet$ fractional urea excretion $>55 \%$; $\bullet$ fluid restriction causing improvement of hyponatremia - no improvement of hyponatremia after $0.9 \%$ saline infusion. 4,5

The different rates of vasopressin secretion, probably denotes different prevalence of SIADH occurring with cancer, for different malignancies, which is of significance, but does not require continuous monitoring. It is noted, patients with malignancy associated SIADH had hyponatremia at follow-up done at short intervals, and irrespective of the management they were given. ${ }^{5}$

In this patient, hyponatremia was associated with decreased serum osmolality and inappropriately increased urine osmolality, natriuresis, absence of renal, cardiac and hepatic disease, normal thyroid function, thus, a classical feature of SIADH and meningioma was tenable. Hyponatremia due to SIADH in a patient with meningioma is thought to result from the mechanical pressure on the hypothalamus and pituitary glands by the compressing mass. ${ }^{4}$

\section{CONCLUSIONS}

In conclusion SIADH, a rare metabolic manifestation of meningioma should be considered whenever hyponatremia is considered especially in the elderly with altered sensorium, as the mortality is higher in euvolemic hyponatremia.

Financial or other competing interests: None.

Disclosure forms provided by the authors are available with the full text of this article at jemds.com.

\section{REFERENCES}

[1] Shepshelovich D, Leibovitch C, Klein A, et al. The syndrome of inappropriate antidiuretic hormone secretion: distribution and characterisation according to etiologies. Eur J Intern Med 2015;26(10):819-24

[2] Verbalis JG, Goldsmith SR, Greenberg A, et al. Diagnosis, evaluation and treatment of hyponatremia: expert panel recommendations. Am J Med 2013;126(10 Suppl 1):S1S42.

[3] Ellison DH, Berl T. The syndrome of inappropriate antidiuresis. N Engl J Med 2007;356(20):2064-72.

[4] Sunil K, Bhayani P, Hathi D, et al. Hyponatremia initial presenting feature of normal pressure hydrocephalus in elderly patient: a rare case report. Journal of Gerontology and Geriatrics 2018;66:156-7.

[5] Lomte S, Jalgaonkar P, Kumar S, et al. Clinical profile of hyponatraemia in a tertiary care hospital. J Evid Based Med Healthc 2017;4(60):3607-13. 